\title{
AICAR Induces Apoptosis and Inhibits Migration and Invasion in Prostate Cancer Cells Through an AMPK/mTOR-Dependent Pathway
}

\author{
Chia-Cheng Su ${ }^{1,2,3,+}{ }^{\dagger}$ Kun-Lin Hsieh ${ }^{1,+}{ }^{\dagger}$ Po-Len Liu ${ }^{4}$, Hsin-Chih Yeh ${ }^{5,6,7}$ (), Shu-Pin Huang ${ }^{5,7}$, \\ Shih-Hua Fang ${ }^{8}$, Wei-Chung Cheng ${ }^{9}$, Kuan-Hua Huang ${ }^{1}$, Fang-Yen Chiu ${ }^{2}$, I-Ling Lin ${ }^{10}$, \\ Ming-Yii Huang 11,12,13,* and Chia-Yang Li $2,14, * \mathbb{C}$ \\ 1 Division of Urology, Department of Surgery, Chi-Mei Medical Center, Tainan 71004, Taiwan; \\ s2341438@yahoo.com.tw (C.-C.S.); samlin.hsieh@gmail.com (K.-L.H.); skhsteven@gmail.com (K.-H.H.) \\ 2 Graduate Institute of Medicine, College of Medicine, Kaohsiung Medical University, \\ Kaohsiung 80708, Taiwan; fangyen0210@hotmail.com \\ 3 Department of Senior Citizen Service Management, Chia Nan University of Pharmacy and Science, \\ Tainan 71710, Taiwan \\ 4 Department of Respiratory Therapy, College of Medicine, Kaohsiung Medical University, \\ Kaohsiung 80708, Taiwan; kisa@kmu.edu.tw \\ 5 Department of Urology, School of Medicine, College of Medicine, Kaohsiung Medical University, Kaohsiung \\ 80708, Taiwan; patrick1201.tw@yahoo.com.tw (H.-C.Y.); shpihu73@gmail.com (S.-P.H.) \\ 6 Department of Urology, Kaohsiung Municipal Ta-Tung Hospital, Kaohsiung 80145, Taiwan \\ 7 Department of Urology, Kaohsiung Medical University Hospital, Kaohsiung Medical University, \\ Kaohsiung 80708, Taiwan \\ 8 Institute of Athletics, National Taiwan University of Sport, Taichung 40404, Taiwan; shfang@ntupes.edu.tw \\ 9 Graduate Institute of Biomedical Sciences, and Research Center for Tumor Medical Science, \\ China Medical University, Taichung 40402, Taiwan; cwc0702@gmail.com \\ 10 Department of Medical Laboratory Science and Biotechnology, College of Health Sciences, \\ Kaohsiung Medical University, Kaohsiung 80708, Taiwan; linili@kmu.edu.tw \\ 11 Department of Radiation Oncology, Kaohsiung Medical University Hospital, Kaohsiung 80756, Taiwan \\ 13 Department of Radiation Oncology, College of Medicine, and Center for Biomarkers and Biotech Drugs, \\ Kaohsiung Medical University, Kaohsiung 80708, Taiwan \\ 14 Center for Infectious Disease and Cancer Research, Kaohsiung Medical University, Kaohsiung 80708, Taiwan \\ * Correspondence: miyihu@gmail.com (M.-Y.H.); chiayangli@kmu.edu.tw (C.-Y.L.); \\ Tel.: +886-7-3121101 (ext. 7122) (M.-Y.H.); +886-7-3121101 (ext. 2759-425) (C.-Y.L.) \\ + These authors contributed equally to this work.
}

Received: 8 March 2019; Accepted: 29 March 2019; Published: 3 April 2019

check for updates

\begin{abstract}
Current clinical challenges of prostate cancer management are to restrict tumor growth and prohibit metastasis. AICAR (5-aminoimidazole-4-carbox-amide-1- $\beta$-D-ribofuranoside), an AMP-activated protein kinase (AMPK) agonist, has demonstrated antitumor activities for several types of cancers. However, the activity of AICAR on the cell growth and metastasis of prostate cancer has not been extensively studied. Herein we examine the effects of AICAR on the cell growth and metastasis of prostate cancer cells. Cell growth was performed by MTT assay and soft agar assay; cell apoptosis was examined by Annexin V/propidium iodide (PI) staining and poly ADP ribose polymerase (PARP) cleavage western blot, while cell migration and invasion were evaluated by wound-healing assay and transwell assay respectively. Epithelial-mesenchymal transition (EMT)-related protein expression and AMPK/mTOR-dependent signaling axis were analyzed by western blot. In addition, we also tested the effect of AICAR on the chemosensitivity to docetaxel using MTT assay. Our results indicated that AICAR inhibits cell growth in prostate cancer cells, but not in non-cancerous prostate cells. In addition, our results demonstrated that AICAR induces apoptosis, attenuates transforming growth factor (TGF)- $\beta$-induced cell migration, invasion and EMT-related protein expression, and enhances the chemosensitivity to docetaxel in
\end{abstract}


prostate cancer cells through regulating the AMPK/mTOR-dependent pathway. These findings support AICAR as a potential therapeutic agent for the treatment of prostate cancer.

Keywords: AICAR; AMPK; prostate cancer; metastasis; chemosensitivity

\section{Introduction}

Prostate cancer is the most common cancer and the second leading cause of cancer-related death among men in the United States [1]. Current treatment options for prostate cancer include surgery, hormonal therapy, chemotherapy, radiation therapy, radiofrequency ablation, high-intensity focused ultrasound, cryotherapy, and cancer vaccine [2,3]. Androgen deprivation therapy (ADT) by surgical or chemical castration has been the mainstay of treatment for advanced prostate cancer in the past few decades. However, the majority of androgen-sensitive prostate cancer patients will eventually develop resistance to ADT within 1 to 3 years and the disease will become androgen-independent [4]. Docetaxel chemotherapy was administered to patients with metastatic castration-resistant prostate cancer (CRPC) for a decade, and demonstrated ability to improve the median overall survival by around 3 months $[5,6]$. Currently, there is no effective therapy for recurrent prostate cancer; hence, a novel therapeutic method for CRPC is needed.

CRPC is often chartered by the reactivation of the androgen receptor (AR) transcriptional activity with castrate levels of androgens because of amplification of AR gene and AR mutation $[7,8]$. Although AR is the classical target for prostate cancer prevention and treatment, several recent studies indicated that estrogen and estrogen receptor (ER) have also been implicated in prostate cancer development, prostate cancer cell stemness, and tumor progression [8,9]. There are two classes of ER, $\mathrm{ER} \alpha$, and $\mathrm{ER} \beta$, which exhibit opposite roles in the progression of prostate cancer. ER $\alpha$ is considered as an oncogene, which mediates the detrimental effects of estrogen including proliferation, inflammation, and carcinogenesis [8], whereas, ER $\beta$ acts as an anti-oncogenic role through protecting prostatic transformation, preventing proliferation and regulating DNA methylation [8]. Therefore, targeting ER might be an effectively strategy to limit the growth and spread of prostate cancer.

The $5^{\prime}$-adenosine monophosphate (AMP)-activated protein kinase (AMPK) is activated by increases in cellular ATP/AMP ratio and plays an important role in regulating glycolytic activity and maintaining energy balance at both cellular and whole body levels [10]. Previous studies indicated that AMPK exhibits intricate relations with other energy/metabolite sensor pathways (e.g., SIRT1, Akt, mTOR, and PARPs) and acts in a coordinated fashion with these [11-15]. AMPK induction leads to enhanced mitochondrial oxidation and mitochondrial biogenesis that have been shown to exert anti-Warburg and anti-proliferative effects in several types of cancers, such as leukemia [16], breast cancer [17], pancreatic cancer [18], hepatocellular carcinoma [19], and prostate cancer [20]. These findings suggest that AMPK activation might be used beneficially for cancer treatment.

The adenoside analog compound, 5-aminoimidazole-4-carbox-amide-1- $\beta$-D-ribofuranoside (AICAR), is intracellularly converted by adenosine kinase to the non-phosphorylated derivative amino-imidazolecarboxamide ribonucleotide (ZMP), an analogue of AMP, which activates AMPK [21]. Therefore, AICAR is often used as an activator of AMPK [22] to modulate cellular energy homeostasis, although AMPK-independent effects have also been proposed [23,24]. A previous study indicated that AICAR inhibited the growth of androgen-independent (DU145, PC3) and androgen-sensitive (LNCaP) cells after four days of treatment [25]. In addition, AICAR inhibited two key enzymes involved in protein synthesis, mTOR and p70S6K, and blocked the ability of the androgen R1881 (methyltrienolone; a synthetic androgen agonist) to increase cell growth and the expression of two enzymes for de novo fatty acid synthesis, acetyl CoA carboxylase and fatty acid synthase, in the LNCaP cells [25]. A current study showed that AICAR induced AMPK-independent programmed necrosis in prostate cancer cells [26]. 
Collectively, these studies indicate that AICAR has potential in inhibiting the growth of prostate cancer. However, the effect of AICAR on the apoptosis and migration of prostate cancer remains unclear. In the present study, we evaluated the effects of AICAR on apoptotic activity, migration, invasion and chemosensitivity through AMPK phosphorylation in human prostate cancer cells.

\section{Results}

\subsection{AICAR Inhibits Cell Growth in Prostate Cancer Cells, but Not in Non-Cancerous Prostate Cells}

To examine the effect of AICAR on the growth of non-cancerous or cancerous prostate cells, cells were treated with various concentrations $(0,0.5,1$, and $3 \mathrm{mM})$ of AICAR for $24 \mathrm{~h}$. Cell viability was analyzed by MTT assay. The experimental results showed that AICAR did not inhibit the growth of non-cancerous prostate cells, PNT1A and BPH1 cells, as well as having pro-survival effect in RWPE-1 cells (Figure 1A), although we found that AICAR had inhibitory effects on the cell growth in cancerous prostate cells, especially in $22 \mathrm{Rv} 1$ and PC 3 cells (Figure 1B). A previous study also showed that AICAR inhibits the growth of androgen-independent (DU145, PC3) and androgen-sensitive (LNCaP) cells [25]; however, the effect of AICAR on 22Rv1 cells has not been examined previously. The following experiments used 22Rv1 cells to explore the inhibitory effect of AICAR in prostate cancer. To examine whether AICAR affects the colony growth under anchorage-independent conditions, 22Rv1 cells were treated with different concentrations $(0,0.25,0.5$ and $1 \mathrm{mM})$ of AICAR and then a soft agar assay was conducted. The experimental results showed that AICAR suppressed growth of 22Rv1 cells in soft agar in a dose-dependent manner (Figure 2).

(A)
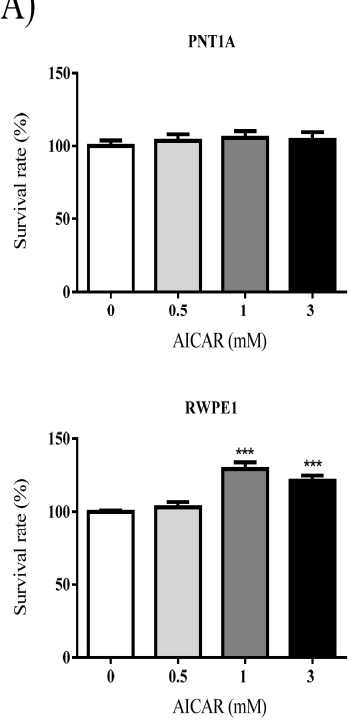

BPH1

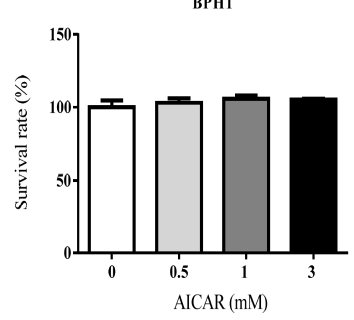

(B)
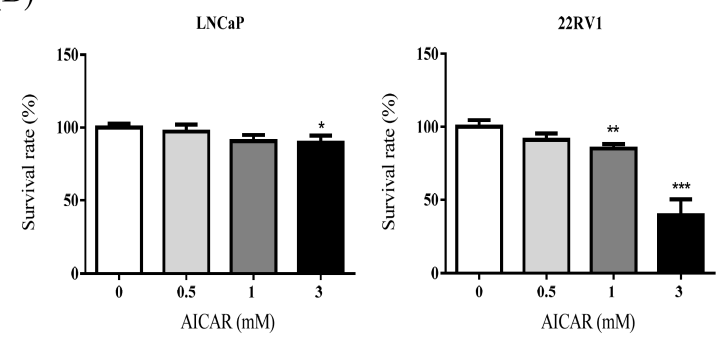

C4-2

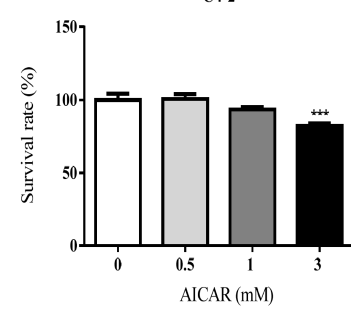

$\mathrm{PC} 3$

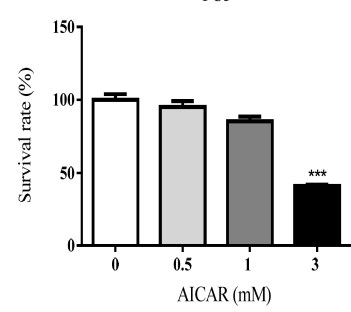

Figure 1. The effect of AICAR (5-aminoimidazole-4-carbox-amide-1- $\beta$-D-ribofuranoside) on the growth of non-cancerous or cancerous prostate cells. (A) non-cancerous or (B) cancerous prostate cells were treated with different concentrations of AICAR for $24 \mathrm{~h}$. The cell viability was measured by the MTT assay. Data are represented as means \pm SD of triplicate values and statistical significance was determined using the Student's t-test $\left({ }^{*} p<0.05 ;{ }^{* *} p<0.01\right.$; $\left.{ }^{* * *} p<0.001\right)$. 
(A) $\operatorname{AICAR}(\mathrm{mM})$

0

0.25
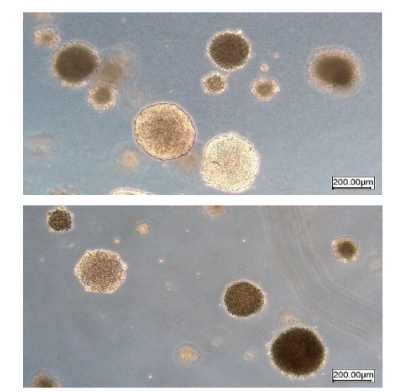

0.5
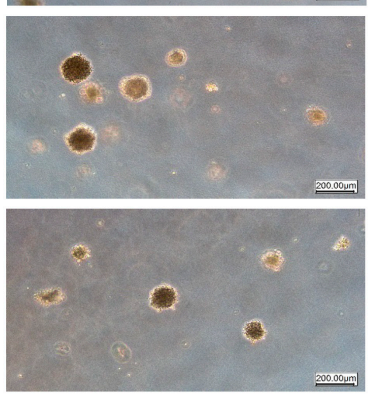

(B)
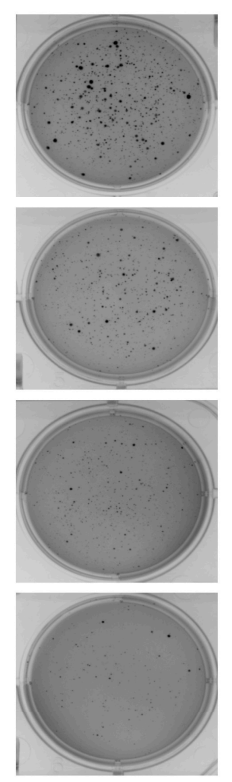

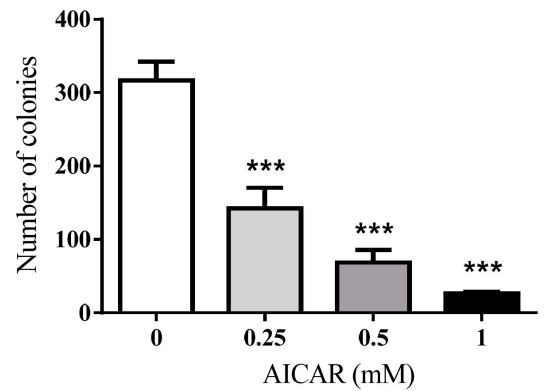

$(50 \mathrm{x})$

Figure 2. The effect of AICAR on growth under anchorage-independent conditions of prostate cancer cells. 22Rv1 cells were treated with different concentrations of AICAR, and then grown in soft agar, an anchorage-independent condition, for 3 weeks. (A) Colonies were stained with crystal violet and captured using the Bio-Rad ChemiDoc XRS+ system (Hercules, CA, USA). (B) Data are quantified and represented as means $\pm \mathrm{SD}$ of triplicate values and statistical significance was determined using the Student's t-test $(* * * 00.001)$.

\subsection{AICAR Induces Apoptosis in Prostate Cancer Cells}

To further test whether AICAR induces apoptosis in prostate cancer cells, 22Rv1 cells were treated with various concentrations $(0,0.5,1$, and $3 \mathrm{mM})$ of AICAR for $24 \mathrm{hr}$. Apoptotic cells were detected with Annexin V/PI staining using flow cytometry. Our results demonstrated that AICAR induced apoptosis (Figure 3A). Poly(ADP-ribose) polymerase (PARP) is a nuclear enzyme involved in DNA repair, which is cleaved by caspase-3 during apoptosis [27]. We further examined whether AICAR affects the expression of PARP in 22Rv1 cells. As shown in Figure 3B, AICAR increased the expression of cleaved PARP, an apoptosis marker, in 22Rv1 cells. In addition, we also examined the activity of caspase $3 / 7$ using a luminescent substrate-based assay. Our results indicated that AICAR increased the activity of caspase 3/7 in 22Rv1 cells (Figure 3C). 
(A)
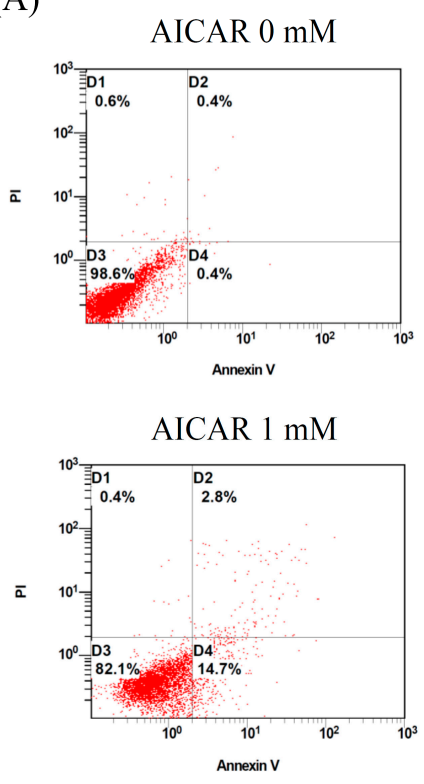
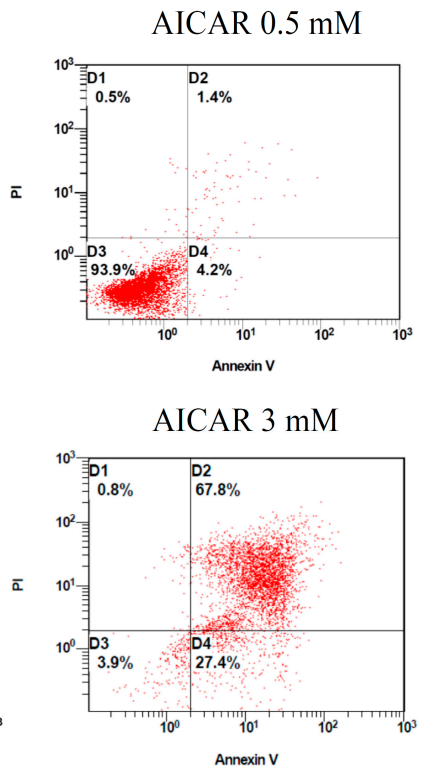

(B)
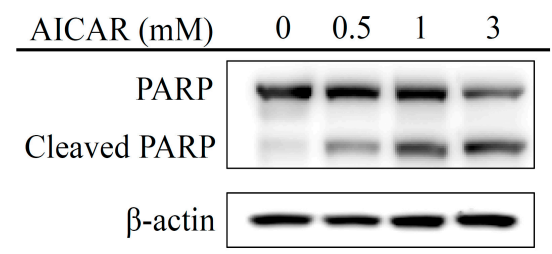

(C)

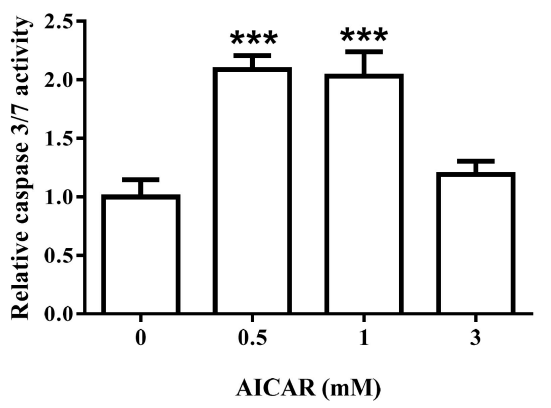

Figure 3. Effect of AICAR on the apoptosis in 22Rv1 prostate cancer cells. Cells were incubated with different concentrations of AICAR for $24 \mathrm{~h}$. (A) Cells were collected, stained with Annexin V and propidium iodide (PI), and analyzed by flow cytometry. Data are representative of at least three independent experiments with similar results. (B) The expression of Poly(ADP-ribose) polymerase (PARP) was determined by western blot. Actin was used as a loading control in western blot. (C) Cellular caspase 3/7 activities were analyzed with caspase-glo assay kit. Data are represented as means \pm SD of triplicate values and statistical significance was determined using the Student's t-test $(* * *<0.001)$.

2.3. AICAR Inhibits Transforming Growth Factor- $\beta$ (TGF- $\beta$ )-Induced Epithelial to Mesenchymal Transition (EMT) and Attenuates TGF- $\beta$-Induced Migration and Invasion Activities

TGF- $\beta$ signaling is well known as a key regulator of many biological processes in prostate cancer including inducing EMT, migration and metastasis [28]. To examine whether AICAR affects TGF- $\beta$-induced EMT, migration, and invasion activities in prostate cancer cells, $22 \mathrm{Rv} 1$ cells were treated with $5 \mathrm{ng} / \mathrm{mL}$ TGF- $\beta$ and various concentrations $(0,0.25$, and $0.5 \mathrm{mM})$ of AICAR. The expression of EMT-related proteins was analyzed using western blot. As shown in Figure 4A, AICAR inhibited TGF- $\beta$-induced EMT through inhibiting the expression of mesenchymal marker, $\mathrm{N}$-cadherin, and enhancing the expression of epithelial marker, E-cadherin. Cell migration and invasion were performed by wound-healing assay and Matrigel transwell assay respectively. Our results showed that AICAR significantly inhibited TGF- $\beta$-induced migration (Figure 4B,C) and invasion (Figure 4D). 
(A)

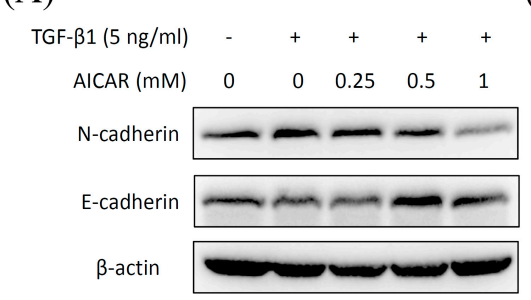

(B)

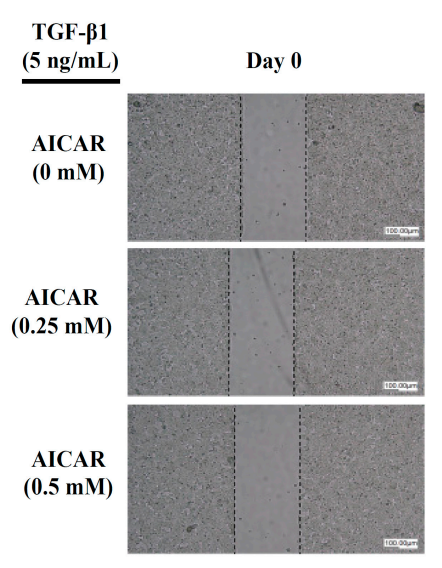

(C)

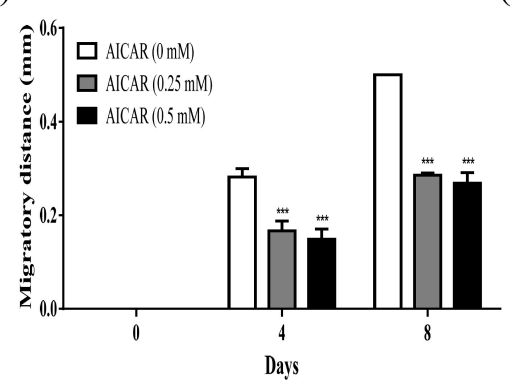

Day 4

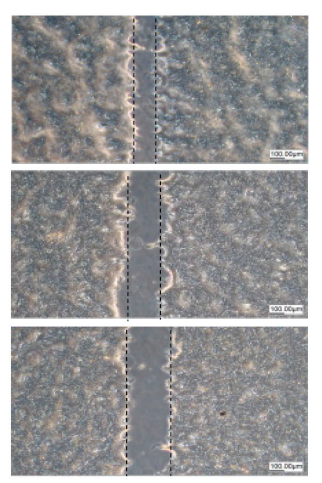

(D)

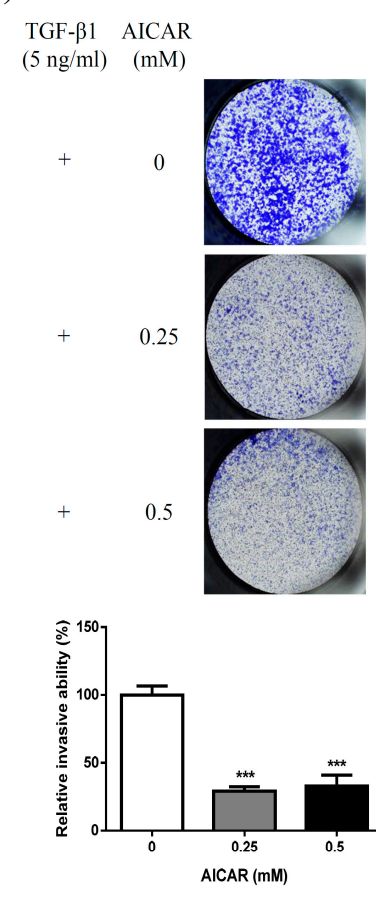

Figure 4. Effect of AICAR on transforming growth factor- $\beta$ (TGF)- $\beta$-induced epithelial to mesenchymal transition (EMT), migration, and invasion in 22Rv1 prostate cancer cells. (A) Cells were treated with $5 \mathrm{ng} / \mathrm{mL}$ TGF- $\beta 1$ and different concentrations of AICAR for $72 \mathrm{~h}$. The expression of N-cadherin and E-cadherin was analyzed by western blot. Actin was used as a loading control in western blot. The western blotting results are representative of results obtained in three separate experiments. (B) Cells were seeded in SPLScarTM Block overnight, the block was then removed, and cells were treated with $5 \mathrm{ng} / \mathrm{mL}$ TGF- $\beta 1$ and different concentrations of AICAR. Cell migration was monitored under a phase-contrast microscope. Data are representative of at least three independent experiments with similar results. (C) The migratory distance was calculated. (D) Cells were treated with $5 \mathrm{ng} / \mathrm{mL}$ TGF- $\beta 1$ and different concentrations of AICAR, then seeded into Matrigel transwell inserts for 5 days. The invaded cells were quantified using crystal violet staining. Data are represented as means \pm SD of triplicate values and statistical significance was determined using the Student's $t$-test $\left({ }^{*} p<0.05 ; * * 0<0.01 ; * * * 0.001\right)$.

\subsection{AICAR Exhibits Synergistic Effect with Docetaxel Treatment}

To examine whether AICAR affects the therapeutic efficiency of docetaxel-based therapy in prostate cancer, $22 \mathrm{Rv} 1$ cells were treated with various doses $(0,0.5$ and $1 \mathrm{mM})$ of AICAR in the presence or absence of docetaxel for $24 \mathrm{~h}$. Cell survival was analyzed by MTT assay. As shown in Figure 5, AICAR exhibited synergistic effect with docetaxel treatment in prostate cancer cells.

\subsection{AICAR Inhibits the Growth of Prostate Cancer Cells Through Activating an Ampk/Mtor-Dependent Pathway}

AMPK is an upstream regulator in modulating the activation of TSC1 and TSC2 which consequently inhibits cell survival and proliferation via repressed mTOR-p70S6K-MYC signaling pathway $[29,30]$. To investigate the mechanism of action of AICAR in regulating the activity of the AMPK-dependent pathway, 22Rv1 cells were treated with different concentrations $(0,0.5,1$, and $3 \mathrm{mM})$ of AICAR. The expression of phospho-AMPK, AMPK, TSC-1, TSC-2, mTOR, phospho-p70S6K, p70S6K, and MYC was analyzed by western blot. As shown in Figure 6A, AICAR promoted the phosphorylation of AMPK in 22Rv1 cells. In addition, our experimental results showed that AICAR enhanced the expression of TSC1 and TSC2 (Figure 6B), whereas AICAR reduced the expression of mTOR and MYC 
as well as decreased the phosphorylation of p70S6K (Figure 6C). These results suggest that AICAR inhibits the growth of prostate cancer cells through an AMPK/mTOR-dependent pathway (Figure 6D).

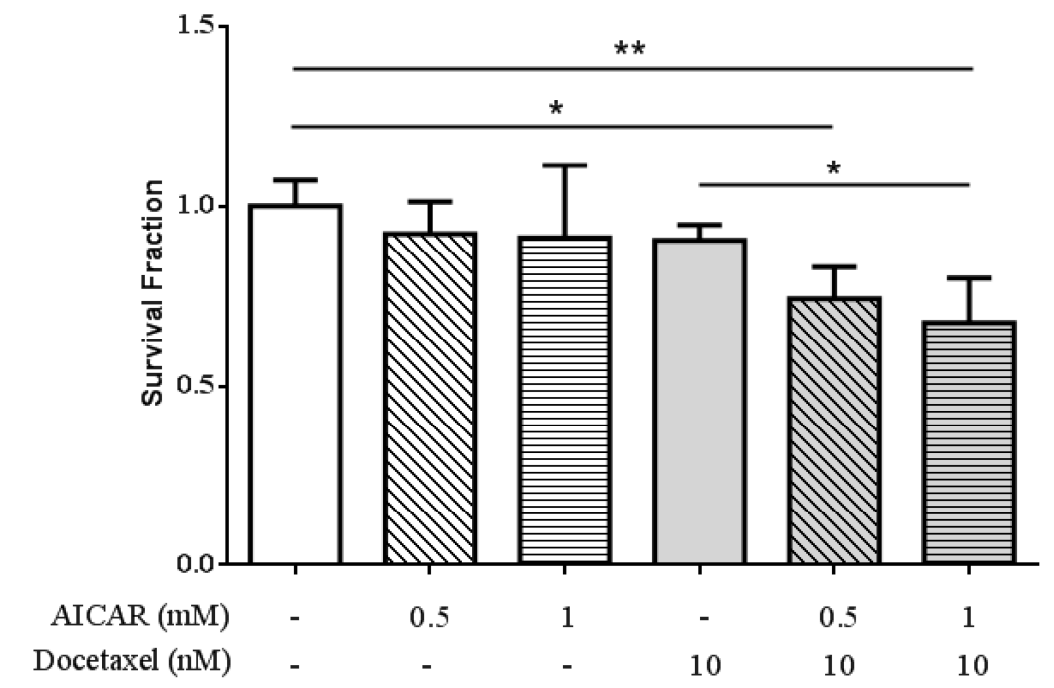

Figure 5. Effect of AICAR on the sensitivity of $22 \mathrm{Rv} 1$ prostate cancer cells to docetaxel treatment. Cells were treated with different concentrations of AICAR in the presence or absence of docetaxel for $24 \mathrm{~h}$. The cell viability was measured by the MTT assay. Data are represented as means \pm SD of triplicate values and statistical significance was determined using the Student's $t$-test $\left({ }^{*} p<0.05 ;{ }^{* *} p<0.01\right)$.

(A)

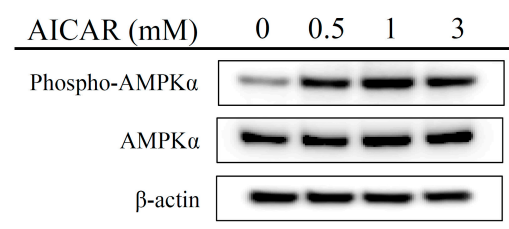

(B)

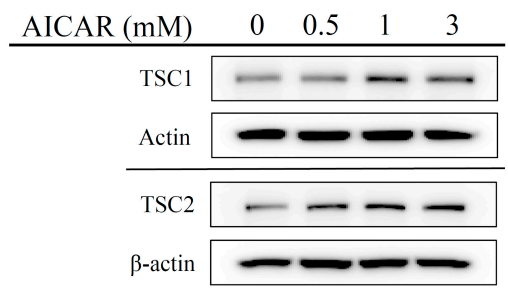

(C)

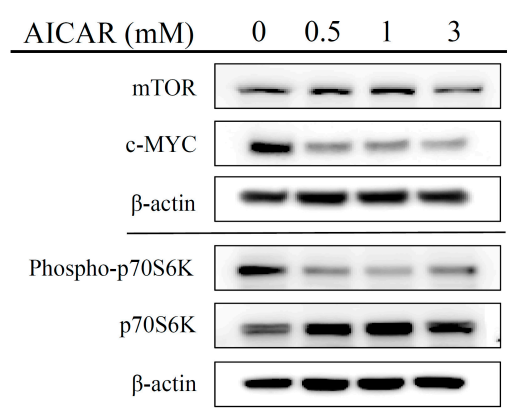

(D)

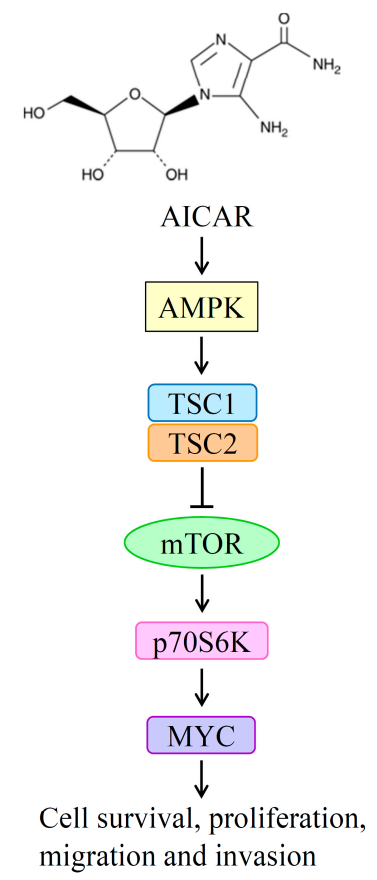

Figure 6. Effect of AICAR on the AMPK/mTOR-dependent pathway in 22Rv1 prostate cancer cells. Cells were treated with different concentrations of AICAR for $2 \mathrm{~h}$. The expression of (A) phospho-AMPK, AMPK, (B) TSC1 and TSC2 was examined by western blot. Cells were treated with different concentrations of AICAR for $6 \mathrm{~h}$. (C) The expression of mTOR, cMYC, phosphor-p70S6K and p70S6K was determined by western blot. Actin was used as a loading control in western blot. The western blotting results are representative of results obtained in three separate experiments. (D) Proposed mechanism of the anticancer effects induced by AICAR in 22Rv1 prostate cancer cells. 


\section{Discussion}

AMPK is an important regulator of cellular energy homeostasis [31]. Several agents have been demonstrated to activate AMPK, including metformin and phenformin, which increase the AMP:ATP ratio, the nucleoside AICAR which is metabolized to an AMP mimetic, and A769662, which is a direct activator of AMPK [32]. Although a number of studies have indicated an anti-cancer role for AMPK $[14,16,17,19,20,33,34]$, the effect of an AMPK activator, AICAR, in regulating the pathological processes of prostate cancer, remains enigmatic. Previous studies have shown that AICAR could exert cytotoxic effect on cancer cells via AMPK-dependent and/or MPK-independent mechanisms $[21,33,35,36]$; however, the cytotoxic effect on prostate cancer remains controversial. Guo et al. indicated that AICAR induces programmed necrosis, but not apoptosis, in prostate cancer cells, LNCaP, PC-3, and PC-82 cells through AMPK-independent pathways [26]. In contrast, Sauer et al. demonstrated that AICAR induces apoptosis of DU-145 prostate cancer cells through the AMPK/mTOR-dependent signaling pathway [21]. Our experimental results indicated that AICAR inhibits the growth of cancerous prostate cells, but not non-cancerous prostate cells. Furthermore, we found that AICAR induces apoptosis, but not necrosis, in 22Rv1 prostate cancer cells. These results point out that AICAR inhibits cell growth and induces cell death in different prostate cancer cell lines probably through different mechanisms. In addition, AMPK is an upstream regulator in modulating the activation of TSC1 and TSC2, which consequently inhibits cell survival and proliferation via repressed mTOR-p70S6K-MYC signaling pathway $[29,30]$. Our experimental results showed that AICAR enhances the expression of TSC1 and TSC2, whereas it reduces the expression of mTOR and MYC as well as decreases the phosphorylation of p70S6K in $22 \mathrm{Rv} 1$ prostate cancer cells. We suggest that AICAR induces apoptosis and inhibits migration of prostate cancer cells through an AMPK/mTOR-dependent pathway.

Cancer metastasis is the major cause of morbidity and mortality; approximately $80 \%$ of the men who have expired from prostate cancer possessed bone metastases [37]. Our experimental results indicated that AICAR suppresses TGF- $\beta$-induced cell migration and invasion as well as inhibits TGF- $\beta$-induced EMT by decreasing the expression of a mesenchymal-related marker, $\mathrm{N}$-cadherin and enhancing the expression of an epithelial-related marker, E-cadherin in 22Rv1 prostate cancer cells. A recent study also indicated that treatment of AICAR suppresses migration and invasion in PC 3 and PC3M prostate cancer cells [20]. These results imply that AICAR might have potential in inhibiting the metastatic activity in prostate cancer.

Previous studies have indicated that ER mediated signaling axis controls prostate cancer growth and progression. Ma et al. indicated that metformin enhances tamoxifen-mediated tumor growth inhibition in ER-positive breast carcinoma [38]. Zhang et al. demonstrated that Metformin significantly decreased E2-stimulated cell proliferation; an effect that was rescued in the presence of compound C (an AMPK inhibitor) [39]. Moreover, metformin significantly inhibited ER $\alpha$ expression while increasing ER $\beta$ expression, whereas treatment with compound $C$ reversed these effects [39]. These results suggest that the activation of AMPK is associated with ER-mediated signaling axis. Our results showed that AICAR has more inhibitory effect in CRPC cells (22Rv1 and PC3) than androgen-dependent prostate cancer cells (LNCaP and C4-2), suggesting that inhibitory effect of AICAR might be associated with the activity of the ER-mediated signaling axis.

Docetaxel is a first-line treatment for CRPC, which confers survival advantages of approximately 2 months for patients with low overall survival benefit [40]; however, treatment with docetaxel usually causes adverse side effects including hair loss, myelosuppression, neurotoxicity, and diarrhea. Our experimental results showed that AICAR has synergistic effect with docetaxel treatment. These results suggest that AICAR increases the sensitization of prostate cancer cells to docetaxel-induced apoptosis, which might have benefit for reducing toxicity of chemotherapy in prostate cancer patients.

In summary, our experimental results indicated that AICAR inhibits cell growth, induces apoptosis, attenuates cell migration, enhances chemosensitivity to docetaxel, and suppresses the 
activation of the AMPK/mTOR-dependent signaling pathway. These results suggest that AICAR appears as a new potential anticancer agent for treating prostate cancer.

\section{Materials and Methods}

\subsection{Reagents}

RPMI 1640 medium, F-12 medium, penicillin, streptomycin and fetal bovine serum (FBS) were purchased from Gibco-BRL (Life Technologies, Grand Island, NY, USA). AICAR, bovine serum albumin (BSA), phosphate-buffered saline (PBS), RIPA buffer, protease inhibitor cocktail, phosphatase inhibitor cocktail, stripping buffer, thioglycollate medium, and 3-(4,5-dimethylthiazol-2-yl)-2, 5-diphenyl tetrazolium bromide (MTT) were purchased from Sigma Aldrich (St. Louis, MO, USA). AICAR was purchased from Cayman Chemical (Ann Arbor, MI, USA). Alexa Fluor ${ }^{\circledR} 488$ Annexin V/Dead Cell Apoptosis Kit was purchased from Thermo Fisher Scientific (Waltham, MA, USA). BCA protein assay reagent was purchased from Thermo Scientific. For western blotting, rabbit antibodies against human phospho-AMPK, AMPK, MYC, mTOR, PARP, phospho-p70S6K, p70S6K, TSC-1, TSC-2, $\beta$-actin and secondary antibodies were purchased from Cell Signaling (Farmingdale, NY, USA). Mouse antibodies against human N-cadherin and E-cadherin were purchased from BD Biosciences (San Jose, CA, USA). Caspase-Glo 3/7 assay kit was purchased from Promega (Madison, WI, USA). Transforming growth factor-beta 1 was purchased from PeproTech (Rocky Hill, NJ, USA). Docetaxel (Taxothere, $20 \mathrm{mg} / \mathrm{mL}$ ) was obtained from Sanofi Aventis (Berlin, Germany).

\subsection{Cell Culture}

Human normal prostate epithelial cell lines (PNT1A and RWPE1), human benign prostatic hyperplasia epithelial cell line (BPH1), and human prostate cancer cell lines (C4-2 and PC3) were obtained from Professor Jer-Tsong Hsieh (Department of Urology, Southwestern Medical Center, University of Texas, TX, USA). Human prostate cancer cell lines (LNCaP and 22Rv1) were purchased from Bioresource Collection and Research Center (Food Industry Research and Development Institute, Hsinchu, Taiwan). Cells were cultured in RPMI 1640 medium (PNT1A, RWPE1, BPH1, LNCaP, C4-2, and 22Rv1) or F-12 medium (PC3), supplemented with antibiotics (100 U/mL penicillin and $100 \mathrm{U} / \mathrm{mL}$ streptomycin) and $10 \%(v / v)$ FBS in a humidified atmosphere of $5 \% \mathrm{CO}_{2}$ at $37^{\circ} \mathrm{C}$ and passaged every 2-3 days to maintain growth.

\subsection{MTT Assay}

Cells were seeded in a 96-well plate at a concentration of $1 \times 10^{5}$ cells, and were allowed to acclimatize overnight. Cells were treated with various concentrations of the AICAR $(0,0.5,1$, and $3 \mathrm{mM}$ ) for $24 \mathrm{~h}$. Cell viability was measured by the ability of viable cells to reduce MTT to formazan based on the ability of living cells utilized thiazolyl blue and converted it into purple formazan. The concentration of formazan is measured by determining the OD at $570 \mathrm{~nm}$ using a microplate reader (BioTek Instruments, Inc., Winooski, VT, USA), and the results are given as relative percentage to the untreated control. To detect the synergistic effects of AICAR, cells were treated with different doses of the AICAR $(0,0.5$, and $1 \mathrm{mM})$ combined with different doses of docetaxel for 24 and $48 \mathrm{~h}$.

\subsection{Soft Agar Colony Formation Assay}

Noble agar (BD Biosciences, Franklin Lakes, NJ, USA) was dissolved in complete medium and coated with $0.5 \%$ agar solution on the bottom of 6-well plates. After solidifying, top agar medium mixture $(0.3 \%)$ containing $5 \times 10^{3}$ cells was added, and incubated at $37^{\circ} \mathrm{C}$ in a humidified atmosphere of $5 \% \mathrm{CO}_{2}$ for 3 weeks. Colonies were stained with $0.05 \%$ crystal violet- $10 \%$ ethanol in PBS. Photographs of the stained colonies were captured using Bio-Rad ChemiDoc XRS ${ }^{+}$system (Bio-Rad 
Laboratories, Inc., Hercules, CA, USA) and quantified using ImageJ software (National Institutes of Health, Bethesda, MD, USA).

\subsection{Apoptosis Assay}

22Rv1 cells were treated with different doses of $\operatorname{AICAR}(0,0.5,1$, and $3 \mathrm{mM})$ for $24 \mathrm{hr}$. Cells were trypsinized, washed twice by cold PBS, and stained with Alexa Fluor ${ }^{\circledR} 488$ Annexin V and propidium iodide (PI) according to the manufacturer's protocol (Thermo Fisher Scientific). Apoptotic cells were determined using FC500 flow cytometer (Beckman-Coulter, Fullerton, CA, USA). Ten thousand events were collected per sample. Data were analyzed by CXP analysis software (Beckman-Coulter, Fullerton, CA, USA).

\subsection{Western Blot}

Cells were lysed by RIPA buffer with protease inhibitors and phosphatase inhibitors according to the manufacturer's protocol (Sigma Aldrich, St. Louis, MO, USA). The protein concentration was determined using the BCA protein assay reagent according to the manufacturer's instructions (Thermo Scientific, Waltham). Cellular protein extracts were separated by electrophoresis using $8 \%$ SDS polyacrylamide gel and were electroblotted onto PVDF membranes. The membranes were incubated with blocking solution for $1 \mathrm{hr}$ at room temperature, followed by incubation overnight with primary antibodies at $4{ }^{\circ} \mathrm{C}$ (1:1000). Blots were washed three times with Tris-buffered saline/Tween 20 (TBST) and incubated with a 1:5000 dilution of horseradish peroxidase conjugated secondary antibody for $1 \mathrm{~h}$ at room temperature. Blots were again washed three times with TBST and developed using an ECL chemiluminescence substrate (Thermo Scientific). The signals were captured and the band intensities were quantified using Bio-Rad ChemiDoc $\mathrm{XRS}^{+}$system (Bio-Rad Laboratories, Inc.).

\subsection{Caspase 3/7 Activity Assay}

22Rv1 cells were seeded in a 96-well white plate at a concentration of $2.5 \times 10^{4}$ cells, and were allowed to acclimatize overnight. Cells were treated with various concentrations of the $\operatorname{AICAR}(0,0.5$, 1 , and $3 \mathrm{mM}$ ) for $24 \mathrm{~h}$. A total of $100 \mu \mathrm{L}$ Caspase-Glo ${ }^{\circledR} 3 / 7$ reagent was added to each well, gently mixed contents of wells using a plate shaker at 300 500 rpm for $30 \mathrm{~s}$, and then samples were incubated for $30 \mathrm{~min}$ at RT. Enzyme activity is directly proportional to luminescence, which was measured using a luminescence microplate reader (BioTek Instruments, Inc.). Data were normalized relative to the caspase $3 / 7$ activity of cells treated with DMSO alone.

\subsection{Migration Assay}

SPLScarTM Block (SPL life sciences, Korea) was placed in a 24-well plate, seeded $5 \times 10^{4}$ cells in each side of block, and was allowed to acclimatize overnight. The block was removed, the flash medium replaced, and cells were treated with $5 \mathrm{ng} / \mathrm{mL}$ TGF- $\beta 1$ and different concentrations of AICAR. Cell migration was monitored under a phase-contrast microscope and the migratory distance was calculated.

\subsection{Invasion Assay}

24-well hanging inserts with $8 \mu \mathrm{m}$ polyester membrane (Millipore Co., Billerica, MA, USA) were used and coated with Matrigel according to the manufacturer's protocol (BD Biosciences, San Jose, CA, USA). A total of $5 \times 10^{4}$ cells in $200 \mu \mathrm{L}$ serum-free medium containing indicated dosage of TGF- $\beta 1$ and AICAR were seeded into the upper chamber and $600 \mu \mathrm{L}$ complete medium $(10 \%$ FBS as chemo attractants) containing indicated dosage of TGF- $\beta 1$ and AICAR was added in the lower chamber. Following incubation for 5 days, non-invading cells were carefully removed with a cotton swab. The cells that passed through the filter were fixed with $5 \%$ glutaldehyde and stained by $0.1 \%$ crystal violet, then photographed. For the quantification of invasiveness, crystal violet was 
dissolved by $50 \%$ methanol and the absorbance was determined at $570 \mathrm{~nm}$ using a microplate reader (BioTek Instruments, Inc.).

\subsection{Statistical Analysis}

All data are expressed as means $\pm \mathrm{SD}$. Each value is the mean of three independent experiments. Statistical analysis was assessed via Student's $t$-test using IBM SPSS Statistics v.19 (IBM Corp., Armonk, NY, USA), and the significant difference was set at ${ }^{*} p<0.05 ;{ }^{* *} p<0.01 ; * * *<0.001$.

Author Contributions: C.-C.S., K.-L.H., P.-L.L., H.-C.Y. and F.-Y.C. performed the experiments; S.-P.H., S.-H.F., W.-C.C., K.-H.H., K.-Y.H., I-L.L., M.-Y.H. and C.-Y.L. produced the study design; C.-C.S., M.-Y.H. and C.-Y.L. wrote the manuscript. All authors approved the final version of the paper.

Funding: This study was supported by Kaohsiung Medical University Research Foundation (Grant No. KMU-M102001), Kaohsiung Medical University Hospital (Grant No. KMUH107-7R73), China Medical University (Grant No. CMU106-N-05), and Chi-Mei Medical Center, Liouying (CLFHR10613). This study was also supported by the Ministry of Science and Technology, Taiwan, R.O.C. (Grant No. MOST 106-2221-E-039-011-MY3, MOST 107-2320-B-037-019 and MOST 107-2314-B-037-047).

Acknowledgments: In this section you can acknowledge any support given which is not covered by the author contribution or funding sections. This may include administrative and technical support, or donations in kind (e.g., materials used for experiments).

Conflicts of Interest: The authors declare no conflict of interest.

$\begin{array}{ll}\text { Abbreviations } \\ \text { AICAR } & \text { 5-aminoimidazole-4-carbox-amide-1- } \beta \text {-D-ribofuranoside } \\ \text { AMPK } & \text { AMP-activated protein kinase } \\ \text { ADT } & \text { Androgen deprivation therapy } \\ \text { CRPC } & \text { Castration-resistant prostate cancer } \\ \text { AR } & \text { Androgen receptor } \\ \text { ER } & \text { Estrogen receptor } \\ \text { FBS } & \text { Fetal bovine serum } \\ \text { BSA } & \text { Bovine serum albumin } \\ \text { PBS } & \text { Phosphate-buffered saline } \\ \text { MTT } & \text { 3-(4,5-dimethylthiazol-2-yl)-2,5-diphenyl tetrazolium bromide } \\ \text { PI } & \text { Propidium iodide } \\ \text { TBST } & \text { Tris-buffered saline/Tween 20 } \\ \text { PARP } & \text { Poly(ADP-ribose) polymerase } \\ \text { TGF- } \beta & \text { Transforming growth factor-beta } \\ \text { EMT } & \text { Epithelial to mesenchymal transition }\end{array}$

\section{References}

1. Siegel, R.L.; Miller, K.D.; Jemal, A. Cancer statistics, 2018. CA Cancer J. Clin. 2018, 68, 7-30. [CrossRef] [PubMed]

2. Litwin, M.S.; Tan, H.J. The diagnosis and treatment of prostate cancer: A review. JAMA 2017, 317, 2532-2542. [CrossRef] [PubMed]

3. Marshall, S.; Taneja, S. Focal therapy for prostate cancer: The current status. Prostate Int. 2015, 3, 35-41. [CrossRef] [PubMed]

4. Asmane, I.; Ceraline, J.; Duclos, B.; Rob, L.; Litique, V.; Barthelemy, P.; Bergerat, J.P.; Dufour, P.; Kurtz, J.E. New strategies for medical management of castration-resistant prostate cancer. Oncology 2011, 80, 1-11. [CrossRef] [PubMed]

5. Petrylak, D.P.; Tangen, C.M.; Hussain, M.H.; Lara, P.N., Jr.; Jones, J.A.; Taplin, M.E.; Burch, P.A.; Berry, D.; Moinpour, C.; Kohli, M.; et al. Docetaxel and estramustine compared with mitoxantrone and prednisone for advanced refractory prostate cancer. N. Engl. J. Med. 2004, 351, 1513-1520. [CrossRef] [PubMed] 
6. Tannock, I.F.; de Wit, R.; Berry, W.R.; Horti, J.; Pluzanska, A.; Chi, K.N.; Oudard, S.; Théodore, C.; James, N.D.; Turesson, I.; et al. Docetaxel plus prednisone or mitoxantrone plus prednisone for advanced prostate cancer. N. Engl. J. Med. 2004, 351, 1502-1512. [CrossRef] [PubMed]

7. Nadiminty, N.; Gao, A.C. Mechanisms of persistent activation of the androgen receptor in CRPC: Recent advances and future perspectives. World J. Urol. 2012, 30, 287-295. [CrossRef] [PubMed]

8. Di Zazzo, E.; Galasso, G.; Giovannelli, P.; Di Donato, M.; Castoria, G. Estrogens and their receptors in prostate cancer: Therapeutic implications. Front. Oncol. 2018, 18, 2. [CrossRef] [PubMed]

9. Di Zazzo, E.; Galasso, G.; Giovannelli, P.; di Donato, M.; di Santi, A.; Cernera, G.; Rossi, V.; Abbondanza, C.; Moncharmont, B.; Sinisi, A.A.; et al. Prostate cancer stem cells: The role of androgen and estrogen receptors. Oncotarget 2016, 7, 193-208. [CrossRef] [PubMed]

10. Grahame Hardie, D. Regulation of AMP-activated protein kinase by natural and synthetic activators. Acta Pharm. Sin. B 2016, 6, 1-19. [CrossRef] [PubMed]

11. Bai, P.; Nagy, L.; Fodor, T.; Liaudet, L.; Pacher, P. Poly(ADP-ribose) polymerases as modulators of mitochondrial activity. Trends Endocrinol. Metab. 2015, 26, 75-83. [CrossRef]

12. Hardie, D.G. AMP-activated/SNF1 protein kinases: Conserved guardians of cellular energy. Nat. Rev. Mol. Cell. Biol. 2007, 8, 774-785. [CrossRef]

13. Hsu, P.P.; Sabatini, D.M. Cancer cell metabolism: Warburg and beyond. Cell 2008, 134, 703-707. [CrossRef]

14. Icard, P.; Lincet, H. A global view of the biochemical pathways involved in the regulation of the metabolism of cancer cells. Biochim. Biophys. Acta 2012, 1826, 423-433. [CrossRef] [PubMed]

15. Kroemer, G. Mitochondria in cancer. Oncogene 2006, 25, 4630-4632. [CrossRef] [PubMed]

16. Kishton, R.J.; Barnes, C.E.; Nichols, A.G.; Cohen, S.; Gerriets, V.A.; Siska, P.J.; Macintyre, A.N.; Goraksha-Hicks, P.; de Cubas, A.A.; Liu, T.; et al. AMPK is essential to balance glycolysis and mitochondrial metabolism to control T-ALL cell stress and survival. Cell. Metab. 2016, 23, 649-662. [CrossRef]

17. Fodor, T.; Szanto, M.; Abdul-Rahman, O.; Nagy, L.; Der, A.; Kiss, B.; Bai, P. Combined treatment of MCF-7 cells with AICAR and methotrexate, arrests cell cycle and reverses Warburg metabolism through AMP-activated protein kinase (AMPK) and FOXO1. PLoS ONE 2016, 11, e0150232. [CrossRef] [PubMed]

18. Cheng, X.; Kim, J.Y.; Ghafoory, S.; Duvaci, T.; Rafiee, R.; Theobald, J.; Alborzinia, H.; Holenya, P.; Fredebohm, J.; Merz, K.H.; et al. Methylisoindigo preferentially kills cancer stem cells by interfering cell metabolism via inhibition of LKB1 and activation of AMPK in PDACs. Mol. Oncol. 2016, 10, 806-824. [CrossRef] [PubMed]

19. Park, S.Y.; Lee, Y.K.; Kim, H.J.; Park, O.J.; Kim, Y.M. AMPK interacts with beta-catenin in the regulation of hepatocellular carcinoma cell proliferation and survival with selenium treatment. Oncol. Rep. 2016, 35, 1566-1572. [CrossRef]

20. Choudhury, Y.; Yang, Z.; Ahmad, I.; Nixon, C.; Salt, I.P.; Leung, H.Y. AMP-activated protein kinase (AMPK) as a potential therapeutic target independent of PI3K/Akt signaling in prostate cancer. Oncoscience 2014, 1, 446-456. [CrossRef]

21. Sauer, H.; Engel, S.; Milosevic, N.; Sharifpanah, F.; Wartenberg, M. Activation of AMP-kinase by AICAR induces apoptosis of DU-145 prostate cancer cells through generation of reactive oxygen species and activation of c-Jun N-terminal kinase. Int. J. Oncol. 2012, 40, 501-508. [CrossRef] [PubMed]

22. Fogarty, S.; Hardie, D.G. Development of protein kinase activators: AMPK as a target in metabolic disorders and cancer. Biochim. Biophys. Acta 2010, 1804, 581-591. [CrossRef] [PubMed]

23. Jacobs, R.L.; Lingrell, S.; Dyck, J.R.; Vance, D.E. Inhibition of hepatic phosphatidylcholine synthesis by 5-aminoimidazole-4-carboxamide-1-beta-4-ribofuranoside is independent of AMP-activated protein kinase activation. J. Biol. Chem. 2007, 282, 4516-4523. [CrossRef] [PubMed]

24. Lopez, J.M.; Santidrian, A.F.; Campas, C.; Gil, J. 5-Aminoimidazole-4-carboxamide riboside induces apoptosis in Jurkat cells, but the AMP-activated protein kinase is not involved. Biochem. J. 2003, 370, 1027-1032. [CrossRef] [PubMed]

25. Xiang, X.; Saha, A.K.; Wen, R.; Ruderman, N.B.; Luo, Z. AMP-activated protein kinase activators can inhibit the growth of prostate cancer cells by multiple mechanisms. Biochem. Biophys. Res. Commun. 2004, 321, 161-167. [CrossRef]

26. Guo, F.; Liu, S.Q.; Gao, X.H.; Zhang, L.Y. AICAR induces AMPK-independent programmed necrosis in prostate cancer cells. Biochem. Biophys. Res. Commun. 2016, 474, 277-283. [CrossRef] [PubMed] 
27. Boulares, A.H.; Yakovlev, A.G.; Ivanova, V.; Stoica, B.A.; Wang, G.; Iyer, S.; Smulson, M. Role of poly(ADP-ribose) polymerase (PARP) cleavage in apoptosis. Caspase 3-resistant PARP mutant increases rates of apoptosis in transfected cells. J. Biol. Chem. 1999, 274, 22932-22940. [CrossRef] [PubMed]

28. Barrett, C.S.; Millena, A.C.; Khan, S.A. TGF-beta effects on prostate cancer cell migration and invasion require FosB. Prostate 2017, 77, 72-81. [CrossRef] [PubMed]

29. Williams, T.; Courchet, J.; Viollet, B.; Brenman, J.E.; Polleux, F. AMP-activated protein kinase (AMPK) activity is not required for neuronal development but regulates axogenesis during metabolic stress. Proc. Natl. Acad. Sci. USA 2011, 108, 5849-5854. [CrossRef]

30. Zhou, W.; Marcus, A.I.; Vertino, P.M. Dysregulation of mTOR activity through LKB1 inactivation. Chin. J. Cancer 2013, 32, 427-433. [CrossRef] [PubMed]

31. Khan, A.S.; Frigo, D.E. A spatiotemporal hypothesis for the regulation, role, and targeting of AMPK in prostate cancer. Nat. Rev. Urol. 2017, 14, 164-180. [CrossRef] [PubMed]

32. Hawley, S.A.; Ross, F.A.; Chevtzoff, C.; Green, K.A.; Evans, A.; Fogarty, S.; Towler, M.C.; Brown, L.J.; Ogunbayo, O.A.; Evans, A.M.; et al. Use of cells expressing gamma subunit variants to identify diverse mechanisms of AMPK activation. Cell. Metab. 2010, 11, 554-565. [CrossRef]

33. Chen, L.; Han, F.; Qu, H.; Yan, H.; Ren, L.; Yang, S. Combination therapy with 5-amino-4-imidazolecarboxamide riboside and arsenic trioxide in acute myeloid leukemia cells involving AMPK/TSC2/mTOR pathway. Pharmazie 2013, 68, 117-123. [PubMed]

34. Lee, K.C.; Lin, C.T.; Chang, S.F.; Chen, C.N.; Liu, J.L.; Huang, W.S. Effect of AICAR and 5-Fluorouracil on X-ray Repair, Cross-Complementing Group 1 Expression, and Consequent Cytotoxicity Regulation in Human HCT-116 Colorectal Cancer Cells. Int. J. Mol. Sci. 2017, 18, 2363. [CrossRef] [PubMed]

35. Santidrian, A.F.; Gonzalez-Girones, D.M.; Iglesias-Serret, D.; Coll-Mulet, L.; Cosialls, A.M.; de Frias, M.; Campàs, C.; González-Barca, E.; Alonso, E.; Labi, V.; et al. AICAR induces apoptosis independently of AMPK and p53 through up-regulation of the BH3-only proteins BIM and NOXA in chronic lymphocytic leukemia cells. Blood 2010, 116, 3023-3032. [CrossRef] [PubMed]

36. Su, R.Y.; Chao, Y.; Chen, T.Y.; Huang, D.Y.; Lin, W.W. 5-Aminoimidazole-4-carboxamide riboside sensitizes TRAIL- and TNF $\alpha$-induced cytotoxicity in colon cancer cells through AMP-activated protein kinase signaling. Mol. Cancer Ther. 2007, 6, 1562-1571. [CrossRef] [PubMed]

37. Bubendorf, L.; Schopfer, A.; Wagner, U.; Sauter, G.; Moch, H.; Willi, N.; Gasser, T.C.; Mihatsch, M.J. Metastatic patterns of prostate cancer: An autopsy study of 1,589 patients. Hum. Pathol. 2000, 31, 578-583. [CrossRef] [PubMed]

38. Ma, J.; Guo, Y.; Chen, S.; Zhong, C.; Xue, Y.; Zhang, Y.; Lai, X.; Wei, Y.; Yu, S.; Zhang, J.; et al. Metformin enhances tamoxifen-mediated tumor growth inhibition in ER-positive breast carcinoma. BMC Cancer 2014, 11, 172. [CrossRef] [PubMed]

39. Zhang, J.; Xu, H.; Zhou, X.; Li, Y.; Liu, T.; Yin, X.; Zhang, B. Role of metformin in inhibiting estrogen-induced proliferation and regulating ER $\alpha$ and ER $\beta$ expression in human endometrial cancer cells. Oncol. Lett. 2017, 14, 4949-4956. [CrossRef] [PubMed]

40. Poorthuis, M.H.F.; Vernooij, R.W.M.; van Moorselaar, R.J.A.; de Reijke, T.M. First-line non-cytotoxic therapy in chemotherapy-naive patients with metastatic castration-resistant prostate cancer: A systematic review of 10 randomised clinical trials. BJU Int. 2017, 119, 831-845. [CrossRef]

(C) 2019 by the authors. Licensee MDPI, Basel, Switzerland. This article is an open access article distributed under the terms and conditions of the Creative Commons Attribution (CC BY) license (http://creativecommons.org/licenses/by/4.0/). 